\title{
Norwood procedure: How I do it
}

Mohan Mathew John, MD, and Emmett Dean McKenzie, MD

Video clip is available online.

Feature Editor's Introduction-The Norwood operation for neonates with hypoplastic left heart syndrome (HLHS) is now more than 40 years old. Between 2014 and 2017, a total of 2737 Norwood operations were performed at 105 STS database participant centers. ${ }^{1}$ Despite innumerable surgical modifications and increased understanding and monitoring of the physiological changes during the perioperative period there is still an operative mortality of $15 \%$ to $20 \% \%$ in both individual institutional reports and national database assessments. ${ }^{1}$

In many respects, the Norwood procedure has become the signature complex neonatal cardiac operation by which surgeons and heart centers are assessed and compared. Despite the intensive focus on and public scrutiny of this operation, a very sobering statistic from the Single Ventricle Reconstruction Trial is that at 6 years post-Norwood, only $60 \%$ of HLHS patients are free from death or cardiac transplantation.

The purpose of this Expert Opinion is to provide precise details of one strategy for surgical performance of the Norwood operation. The technique was developed and refined by Dr E. Dean McKenzie, a highly accomplished neonatal cardiac surgeon at one of the top congenital heart surgery programs in the United States.

The steps of the operation are well described in the article, but the video link is the most important part of the opinion piece. The video shows in detail and real time the many clever surgical minutiae required for a successful outcome of this complex operation.

Not only congenital heart surgeons in-training who are learning to perform this operation, but also surgeons who have been performing the operation for many years can

From the Division of Congenital Heart Surgery, Texas Children's Hospital, Houston, Tex

Received for publication Aug 5, 2020; revisions received Aug 5, 2020; accepted for publication Aug 11, 2020; available ahead of print Aug 13, 2020.

Address for reprints: Emmett Dean McKenzie, MD, 6651 Main St, Legacy Tower, 19th Floor, E.1980.23, Houston, TX 77030 (E-mail: edmcken1@texaschildrens. org).

JTCVS Techniques 2020;4:205-7

2666-2507

Copyright (C) 2020 The Authors. Published by Elsevier Inc. on behalf of The American Association for Thoracic Surgery. This is an open access article under the CC BY-NCND license (http://creativecommons.org/licenses/by-nc-nd/4.0/).

https://doi.org/10.1016/j.xjtc.2020.08.026

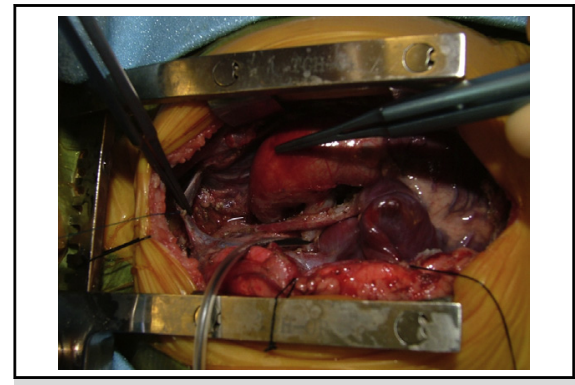

Intraoperative view of hypoplastic left heart syndrome.

CENTRAL MESSAGE

We present our approach to the

Norwood operation with a

modified Blalock-Taussig shunt, accompanied by an edited video.

learn valuable technical lessons from viewing this excellent "How I Do It" expert opinion.

\section{Carl L. Backer, MD}

The Norwood procedure is considered the gold standard for palliation of hypoplastic left heart syndrome (HLHS). Multiple variations in surgical technique have been described in the literature; however, the goals of first-stage palliation remain the same: unobstructed systemic blood flow to the aorta and coronary arteries, a controlled source of pulmonary blood flow, and unobstructed egress of pulmonary venous return. This article, accompanied by an edited video (Video 1), describes our approach to the Norwood operation with a modified Blalock-Taussig (BT) shunt.

The fundamental steps of our technique are as follows: (1) right atrial cannulation; (2) right subclavian artery (RSCA) cannulation via an expanded polytetrafluoroethylene (ePTFE) graft; (3) aortic arch dissection while cooling to $18{ }^{\circ} \mathrm{C}$; (4) Del Nido cardioplegia delivered via an aortic cannula; (5) atrial septectomy under deep hypothermic circulatory arrest; (6) reconstruction of the aortic arch with antegrade cerebral perfusion; (7) amalgamation of the augmented aorta with the pulmonary artery; (8) transfer of arterial cannula to the augmented ascending aorta; and 


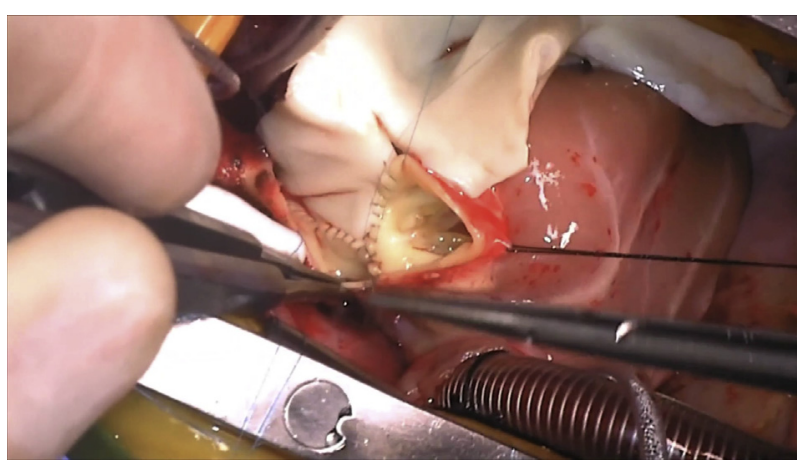

VIDEO 1. Norwood procedure: surgical technique. PDA, patent ductus arteriosus; MPA, main pulmonary artery; RPA, right pulmonary artery; RA, right atrium; RCCA, right common carotid artery; RSCA, right subclavian artery; Innom a., innominate artery; Innom v., innominate vein; deg, degrees; Asc Ao, ascending aorta; Desc Ao, descending aorta; DTA, descending thoracic aorta; BT, Blalock-Taussig. Video available at: https://www.jtcvs.org/article/S2666-2507(20)30401-6/fulltext.

(9) completion of a modified BT shunt using the previously placed ePTFE graft.

\section{ANESTHETIC CONCERNS}

Nasotracheal intubation is preferred to maintain stability of the airway. Transesophageal echocardiography is not used, because there is little information to be gained and the probe hampers dissection of the distal descending thoracic aorta (DTA). A left radial arterial line is preferred, because a right modified BT shunt is created for the source of pulmonary blood flow. Bilateral cerebral near-infrared spectroscopy is used to monitor for adequate neuroprotection. Femoral or jugular venous central lines are avoided because of the high incidence of thrombotic complications. An existing umbilical vein catheter or peripherally inserted central catheter is preferred for central access.

\section{PREPARATION FOR CARDIOPULMONARY BYPASS}

After a full sternotomy and thymectomy, the pericardium is opened. Partial occlusion of the bilateral branch pulmonary arteries is accomplished using fine tourniquets created with 4-0 silk and a 3.5 Fr feeding tube. Homograft patch material for arch reconstruction is chosen and prepared. The ascending aorta, pulmonary root, and ductus arteriosus are then dissected and mobilized. Care is taken to avoid excessive traction on the diminutive ascending aorta during dissection, which may compromise coronary blood flow.

Marking sutures are placed on the native ascending aorta and pulmonary root to facilitate accurate approximation of the aorta and transected pulmonary artery. The marking suture on the main pulmonary artery (MPA) should be inferior to the right pulmonary artery (RPA) origin and directly opposite the small native aorta, so that when the MPA is later transected, the suture remains with the root. A purse- string suture is placed around the pulmonary end of the patent ductus arteriosus (PDA), with care taken to avoid compromising the lumen of the left pulmonary artery (LPA). A tourniquet is passed over the suture for later use.

The innominate artery, right common carotid artery (RCCA), and RSCA are then extensively mobilized, and a marking suture is placed on the inferior aspect of the RSCA to avoid mislocating the incision. Then $100 \mathrm{U} / \mathrm{kg}$ of unfractionated heparin is given i.v., and activated clotting time is assessed (typical reading, 180 to 200 seconds). A $3.5-\mathrm{mm}$ ePTFE graft is standard for infants weighing $>2.5 \mathrm{~kg}$. The graft is beveled at an acute angle to prevent the heel of the anastomosis from distorting the origin of the RSCA. The innominate artery, RCCA, and RSCA are controlled with fine tourniquets described above, and a longitudinal incision is created on the inferior aspect of the RSCA.

Typically, the incision is not carried proximally onto the innominate artery, which allows the native subclavian artery to regulate pulmonary blood flow. A graft-to-artery anastomosis is then created using a 8-0 polypropylene suture. Thrombin-soaked Gelfoam and fibrin sealant are applied to the anastomotic site. The graft is deaired and occluded with a Yasargil clip, and flow is restored to the RCCA and RSCA. Full heparinization is then achieved. The graft is then cannulated and connected to the arterial limb of the cardiopulmonary bypass (CPB) circuit with an interposing stop-cock for subsequent administration of cardioplegia. Under partial occlusion clamp, a generous defect is created in the right atrium for both single atrial cannulation and atrial septectomy. The surgeon should be cognizant of the location and orientation of the tip of the atrial cannula to keep it from traumatizing the atrioventricular node and tricuspid valve. A malleable bullet tip cannula is preferred.

\section{CPB AND SYSTEMIC COOLING}

CPB is initiated once the circuit is completed and the tourniquet around the PDA is snared. Phentolamine 0.1 to $0.3 \mathrm{mg}$ is given, and a full flow of $150 \mathrm{~mL} / \mathrm{kg} / \mathrm{minute}$ is maintained regardless of temperature. $\mathrm{pH}$ stat acid-base management is maintained during cooling, with the temperature slowly decreased to $18{ }^{\circ} \mathrm{C}$ over at least 20 minutes. A decellularized right hemi-pulmonary artery homograft is preferred for arch reconstruction and is tailored into the shape of a sail or shark fin. The patch should not be made too large, which can compromise the LPA, or too long, which tends to create an acute angulation at the distal arch. The patch is folded along the RPA and then cut at a 30-degree angle.

The brachiocephalic arteries and proximal DTA are dissected, and fine tourniquets are placed for later control of the vessels. The MPA is transected immediately above the pulmonary commissures. This leaves excess tissue on the left side of the pulmonary bifurcation that when closed 
primarily in a sagittal plane augments the inherent deficit of tissue near the RPA.

\section{MYOCARDIAL ARREST AND AORTIC ARCH RECONSTRUCTION}

The cardioplegia line is flushed and connected to the stopcock in the arterial limb. CBP flow is reduced as the descending thoracic, left and right subclavian, and left and right common carotid arteries are snared. Then CPB is briefly discontinued, and cardioplegia is administered via the arterial cannula. The venous cannula is removed, and an atrial septectomy is performed, working through the cannulation site. The venous cannula is returned to the right atrium. The innominate artery is snared between the arch and ePTFE graft, and the tourniquets are removed from the RSCA and RCCA. Selective cerebral perfusion is initiated at $50 \mathrm{~mL} / \mathrm{kg} / \mathrm{min}$. The PDA is divided, the DTA is extensively mobilized, and the tourniquet is repositioned as distally as possible. The DTA, lesser curvature of the arch, and ascending aorta are then incised longitudinally, with the ascending incision carried up to the marking suture. All ductal tissue and the posterior ledge are resected, and an end-to-end three-quarter circumference anastomosis is created between the DTA and the arch just beyond the left subclavian artery.

The decellularized pulmonary homograft patch is then used to enlarge the proximal DTA and arch up to the innominate artery. With the patch properly oriented, the posterior aspect of the patch is cut exactly parallel with the opened native ascending aorta, and the patch is secured to the posterior aortic incision. A direct aortopulmonary anastomosis is then created between the transected MPA and the inferior apex of the native aortic incision. This is typically done with running 8-0 polypropylene and requires no more than 5 passes of the needle. The patch is then further tailored to accommodate incorporation of the MPA and is cut at a slight angle cephalad to prevent the patch from being too long. The patch is then sutured to the MPA and, after further tailoring, brought down to the anterior aspect of the native ascending aorta. The anastomosis is then glued using small pieces of Gelfoam soaked in saline to which small amounts of fibrin sealant are applied. Care is taken to avoid spillage of fibrin sealant while on CPB. The heart and arch are deaired through the anterior patch/MPA anastomosis by having the perfusionist briefly partially occlude the venous return. The patch/MPA anastomosis is finalized, and tourniquets are removed from the DTA and brachiocephalic arteries, ending the myocardial arrest. Full flow on CPB is restored, and rewarming is initiated. It is important to relieve any temporary distention of the ventricle if present.

\section{CREATION OF THE MODIFIED BT SHUNT}

An $8 \mathrm{Fr}$ arterial cannula is placed via a small purse-string suture in the neoaorta, deaired, and connected to the arterial limb of the CPB circuit. The pump is turned off briefly while the connection is made. The ePTFE graft is occluded with a Yasargil clip near the RSCA and tailored appropriately. On confirmation that the pulmonary bifurcation and LPA are free from distortion, a longitudinal arteriotomy is created in the RPA as centrally as possible, and a graft to the RPA anastomosis is created. A small amount of flow is allowed through the shunt as the lungs are recruited.

\section{SEPARATION FROM CPB}

During rewarming, continuous ultrafiltration is performed to maintain a hematocrit value of $45 \%$ to $50 \%$. The patient is weaned from CPB on low-dose epinephrine and calcium infusions as the shunt is opened. Once adequate hemodynamics and gas exchange are confirmed, protamine is administered, and a peritoneal dialysis catheter is placed in addition to a tunneled double-lumen catheter in the right atrium via the cannulation site. With the arch anastomosis glued, bleeding is minimal, and in many cases blood products are not required. Milrinone is typically initiated once there is generous blood pressure. The pericardium is approximated, if possible, to facilitate entry into the chest at resternotomy. The sternal incision is closed in approximately $80 \%$ of patients. Peritoneal dialysis is initiated within 4 to 6 hours of admission to the intensive care unit.

\section{Conflict of Interest Statement}

The authors reported no conflicts of interest.

The Journal policy requires editors and reviewers to disclose conflicts of interest and to decline handling or reviewing manuscripts for which they may have a conflict of interest. The editors and reviewers of this article have no conflicts of interest.

\footnotetext{
References

1. Jacobs JP, Mayer JE Jr, Pasquali SK, Tweddell JS, Dearani JA, Jacobs ML, et al. The Society of Thoracic Surgeons Congenital Heart Surgery Database: 2019 update on outcomes and quality. Ann Thorac Surg. 2019; 107:691-704

2. Newburger JW, Sleeper LA, Gaynor JW, Hollenbeck-Pringle D, Frommelt PC Li JS, et al. Transplant-free survival and interventions at 6 years in the SVR trial. Circulation. 2018;137:2246-53.
}

Key Words: hypoplastic left heart syndrome, Norwood procedure 